hep-th/0008081

\title{
The Enhançon and $\mathcal{N}=2$ Gauge Theory/Gravity RG Flows
}

\author{
Nick Evans ${ }^{\natural}$, Clifford V. Johnson ${ }^{\natural}$, Michela Petrini ${ }^{\sharp}$ \\ ${ }^{b}$ Department of Physics, University of Southampton \\ Southampton SO17 1BJ, U.K. \\ ${ }^{\natural}$ Centre for Particle Theory, Department of Mathematical Sciences \\ University of Durham, Durham, DH1 3LE, U.K. \\ \# Theoretical Physics Group, Blackett Laboratories \\ Imperial College, London SW7 2BZ, U.K. \\ n.evans@hep.phys.soton.ac.uk, c.v.johnson@durham.ac.uk, m.petrini@ic.ac.uk
}

\begin{abstract}
We study the family of ten dimensional type IIB supergravity solutions corresponding to renormalisation group flows from $\mathcal{N}=4$ to $\mathcal{N}=2$ supersymmetric $S U(N)$ Yang-Mills theory. Part of the solution set corresponds to a submanifold of the Coulomb branch of the gauge theory, and we use a D3-brane probe to uncover details of this physics. At generic places where supergravity is singular, the smooth physics of the probe yields the correct one-loop form of the effective low energy gauge coupling. The probe becomes tensionless on a ring at finite radius. Supergravity flows which end on this "enhançon" ring correspond to the vacua where extra massless degrees of freedom appear in the gauge theory, and the gauge coupling diverges there. We identify an $S L(2, \mathbb{Z})$ duality action on the enhançon ring which relates the special vacua, and comment on the massless dyons within them. We propose that the supergravity solution inside the enhançon ring should be excised, since the probe's tension is unphysical there.
\end{abstract}




\section{Introduction}

There has been some emphasis on the construction of supergravity solutions which have a dual interpretation as the renormalisation group flow from $\mathcal{N}=4$ supersymmetric pure Yang-Mills theory in the ultraviolet (UV) to $\mathcal{N}=2,1$ or 0 supersymmetric Yang-Mills theories of various sorts, in the infrared (IR) [1]- 18$]$.

The supergravity solutions interpolate between $\mathrm{AdS}_{5} \times S^{5}$, the dual of the $\mathcal{N}=4$ theory $[19,20$, 21], which is at $r=\infty$ (where $r$ is a suitably chosen radial coordinate of $\mathrm{AdS}_{5}$ ), and some much more complex solution in the interior, towards smaller $r$. Such solutions are typically found in 5 dimensional supergravity, and in some cases they can be lifted to 10 dimensional type IIB solutions. They often possess naked curvature singularities at finite values of $r$ 22]. It is clear that some of these singularities are simply not physical, and the whole supergravity solution represents a flow to field theory with pathological behaviour, which presumably would be represented in terms of a pathology of the full type IIB string theory solution. For other singularities, it is to be expected that the full stringy physics will resolve the singular physics into benign phenomena consistent with the dual gauge theory.

The full technology for studying string theory in such backgrounds where there is high curvature and strong Ramond-Ramond fields - is yet to be developed to a point where we can answer all of the questions raised by these issues. However, while we wait for these techniques, we may try to use the tools already at our disposal for clues as to how the physics of such singularities is to be resolved.

In a context slightly different from the AdS/CFT correspondence, supergravity solutions containing the physics of (but not fully dual to or decoupled from) pure $\mathcal{N}=2$ supersymmetric Yang-Mills theory were found to have naked, unphysical singularities [23]. The singular solutions were not 
obtained as RG flows from some smooth supergravity dual of a more symmetric theory, but were deduced from properties of the gauge theory it was desired to represent, combined with knowledge of the parent string theory and the world--volume curvature couplings of the branes involved.

Using various clues and techniques, a method was found by which string theory excises the singular part of the solution in a way that was consistent with the gauge theory physics. This is called the "enhançon mechanism", and simply put, the idea is that the constituent branes making up the supergravity/string solution have expanded to form a spherical shell (called the "enhançon") around the region which appears singular in supergravity. The interior of this shell contains no point-like brane sources at all, and is (to a first approximation) a smooth, flat region of spacetime [23.

A key tool in arriving at this conclusion was the idea of using a single D-brane as a probe of the geometry created by the others. D-branes as probes of the "true" nature of spacetime at short distances and/or at strong string coupling, have been shown to be extremely useful, and when combined with ideas from gauge theory 24 (which lives on the brane's world-volume [25, 26]), form part of a kit of sharp tools for studying the issues at hand 27. 17

In this short note, we would like to turn to the issue of the supergravity "duals" of $\mathcal{N}=2$ supersymmetric Yang-Mills theory again, but now in the context of solutions describing RG flow from the $\mathcal{N}=4$ theory in the presence of appropriate masses. Recently, the full ten dimensional "lift" of the five dimensional supergravity solutions representing the flows [13, 15, 16] has been presented [15]. The point is that, just like in the case of ref. 223, the dual physics is not just that of supergravity, but of the full type IIB string theory, and the idea here is to try to uncover some of the essential details of the crossover between the two.

\footnotetext{
${ }^{1}$ For a collection of pedagogical studies with a focus on these techniques, the reader may wish to have ref. 28 to hand.
} 
The ten dimensional solutions are generically singular in the IR, having naked curvature singularities, while being smooth in the UV, where they are weakly curved, matching onto $\mathrm{AdS}_{5} \times S^{5}$, which is dual to the $\mathcal{N}=4$ supersymmetric $S U(N)$ Yang-Mills theory at large $N$. We introduce a D3-brane probe and study the nature of the effective Lagrangian for moving the D3brane probe in a supersymmetric way in the subspace corresponding to (a part of) the Coulomb branch of the $\mathcal{N}=2$ supersymmetric four dimensional gauge theory. The result has the complementary interpretation as the effective geometry seen by the D3-brane probe as it moves in ten dimensional spacetime, and as the physics of the moduli space of the gauge theory on the constituent branes.

We find that there is an enhançon shell, just as in the prototype cases studied in ref. [23], which in this case is a ring. Here, there is no enhanced gauge symmetry in the parent supergravity, (which was partly responsible for the name "enhançon"), but all of the other key phenomena are present. In summary:

- There is a ring where the tension of the probe brane drops to zero, signalling the last radius where there is any meaning to the constituent branes as localised sources. The branes have spread out into a ring there and the supergravity solution interior to that — which is singularshould be drastically modified by smoothing.

- The full result for the spacetime metric as seen by the probe interpolates smoothly between the simple behaviour at infinity and that of the interior. In gauge theory language this results in a description of the behaviour of the gauge coupling from the UV (where it is constant) to the IR (where it runs logarithmically). 2]

\footnotetext{
${ }^{2}$ Since the work presented in ref. 23] was a study of the pure $\mathcal{N}=2$ gauge theory in isolation, i.e. not connected to another gauge theory by a massive continuous RG flow, the smooth interpolation to the physics of the $\mathcal{N}=4$ gauge theory is not present there.
} 
- Deep in the IR, the coupling of the gauge theory is seen to run logarithmically, and it diverges at the enhançon ring, representing the locus of points on the Coulomb branch where new massless degrees of freedom appear in the gauge theory.

- The enhançon ring itself characterises a family of vacua at which all species of $(p, q)$ dyons can become massless. There is a descendant of the type IIB string theory's $S L(2, \mathbb{Z})$ duality group which acts naturally on and within the vacua, and the existence of the dyons with this action follows from the existence of bound states of type IIB strings. There is a beautiful pattern of relationships between the vacua as one goes around the circle.

It is very encouraging to see the enhançon behaviour appearing so naturally in the supergravity flow arena, and we expect that this technique will find further fruitful application in this context as more ten dimensional supergravity solutions are found.

\section{The Ten Dimensional Solution}

The family of ten dimensional solutions of ref. 15 describing the gravity dual of $\mathcal{N}=4$ supersymmetric $S U(N)$ Yang-Mills theory, broken to $\mathcal{N}=2$ in the IR may be written as:

$$
d s_{10}^{2}=\Omega^{2} d s_{1,4}^{2}+d s_{5}^{2}
$$

for the Einstein metric, where

$$
d s_{1,4}^{2}=e^{2 A(r)}\left(-d t^{2}+d x_{1}^{2}+d x_{2}^{2}+d x_{3}^{2}\right)+d r^{2},
$$

and

$$
d s_{5}^{2}=L^{2} \frac{\Omega^{2}}{\rho^{2}}\left[\frac{d \theta^{2}}{c}+\rho^{6} \cos ^{2} \theta\left(\frac{\sigma_{1}^{2}}{c X_{2}}+\frac{\sigma_{2}^{2}+\sigma_{3}^{2}}{X_{1}}\right)+\sin ^{2} \theta \frac{d \phi^{2}}{X_{2}}\right]
$$


with $c=\cosh (2 \chi)$, and

$$
\begin{aligned}
& \Omega^{2}=\frac{\left(c X_{1} X_{2}\right)^{1 / 4}}{\rho} \\
& X_{1}=\cos ^{2} \theta+\rho^{6} \cosh (2 \chi) \sin ^{2} \theta \\
& X_{2}=\cosh (2 \chi) \cos ^{2} \theta+\rho^{6} \sin ^{2} \theta .
\end{aligned}
$$

The $\sigma_{i}$ are the standard $S U(2)$ left-invariant forms, the sum of the squares of which would give the standard metric on a round three-sphere.

The functions $\rho(r)=e^{\alpha(r)}$ and $\chi(r)$ which appear in the lifted 10 dimensional metric are the 5 dimensional supergravity scalars pertaining to certain operators in the dual gauge theory. There is a one-parameter family of solutions for them, giving a family of supergravity solutions, and correspondingly a slice through a moduli space of $\mathcal{N}=2$ theories in the IR. We will discuss $\rho(r)$ and $\chi(r)$ further shortly.

At $r \rightarrow \infty$, the various functions in the solution have the following asymptotic behaviour:

$$
\rho(r) \rightarrow 1, \chi(r) \rightarrow 0, A(r) \rightarrow r / L,
$$

where $L=\alpha^{1 / 2}\left(2 g_{\mathrm{YM}}^{2} N\right)^{1 / 4}$. Also $g_{\mathrm{YM}}^{2}=2 \pi g_{s}$, here.

It is easily seen that the non-trivial radial dependences of $\rho(r)$ and $\chi(r)$ deform the supergravity solution from $\mathrm{AdS}_{5} \times S^{5}$ at $r=\infty$ where there is an obvious $S O(6)$ symmetry (the round $S^{5}$ is restored), to a spacetime which only has an $S U(2) \times U(1)^{2}$ symmetry, which is manifest in the metric (3).

There are also explicit solutions given in ref.[15] for the dilaton, $\Phi$, the R-R scalar and two-form potential, $C_{(0)}$ and $C_{(2)}$, and the NS-NS two-form potential $B_{(2)}$. In fact, for the subspace of the solutions which we will probe in this paper (see later), the two-form fields are zero, and so we shall not list their full form here.

The fields $\left(\Phi, C_{(0)}\right)$ are gathered into a complex scalar field which we shall denote as $\lambda=C_{(0)}+i e^{-\Phi}$. This is a natural object in the basis where the classical $S L(2, \mathbb{R})$ symmetry of the type IIB supergravity is manifest. However, 
in ref. 15 the more venerable $S U(1,1)$ basis is used, and the supergravity field $B$ given there is related to $\lambda$ as follows:

$$
\lambda=i\left(\frac{1-B}{1+B}\right) \text {. }
$$

The explicit solution is

$$
B=\left[\frac{b^{1 / 4}-b^{-1 / 4}}{b^{1 /}+b^{-1 / 4}}\right] e^{2 i \phi}, \text { where } b \equiv c \frac{X_{1}}{X_{2}} .
$$

In fact, we shall not yet need the explicit form for the dilaton because it disappears from the D3-brane probe action when it is written in Einstein frame, as we shall do in section 4 . The scalar field $C_{(0)}$ couples to $F \wedge F$ on the D3-brane world volume, contributing to the $\theta$-angle in the $\mathcal{N}=2$ effective low energy theory.

We will need the explicit form for the $\mathrm{R}-\mathrm{R}$ four form potential $C_{(4)}$, to which the D3-brane naturally couples. It is: [

$$
C_{(4)}=e^{4 A} \frac{X_{1}}{g_{s} \rho^{2}} d x_{0} \wedge d x_{1} \wedge d x_{2} \wedge d x_{3} .
$$

The radial dependences of the various functions which appear in the solution are given by the $5 d$ supergravity equations of motion, written in the following form (recall that $\rho=\exp (\alpha))$ :

$$
\begin{aligned}
\frac{d \alpha}{d r} & =\frac{g}{6}\left(\frac{1}{\rho^{2}}-\rho^{4} \cosh (2 \chi)\right) \\
\frac{d \chi}{d r} & =-\frac{g}{4} \rho^{4} \sinh (2 \chi) \\
\frac{d A}{d r} & =\frac{g}{3}\left(\frac{1}{\rho^{2}}+\frac{1}{2} \rho^{4} \cosh (2 \chi)\right),
\end{aligned}
$$

\footnotetext{
${ }^{3}$ We noticed that the expression given in equation (4.9) of ref. 15 is not consistent with their equation (3.31), and we assume that it is a typographical error. There is a crucial factor of $\sinh ^{2}(2 \chi)$ missing. Instead of checking the ten dimensional solution by hand, we verify this correction by requiring that the potential seen by the brane probe is constant, as required by supersymmetry. We have also inserted a factor of $g_{s}^{-1}$ to match our standard conventions for the D3-brane charge.
} 
(where $g=2 / L$ ) with the partial result [15, 16]:

$$
\begin{aligned}
e^{A} & =k \frac{\rho^{2}}{\sinh (2 \chi)} \\
\rho^{6} & =\cosh (2 \chi)+\sinh ^{2}(2 \chi)\left(\gamma+\log \left[\frac{\sinh \chi}{\cosh \chi}\right]\right) .
\end{aligned}
$$

Here, $k$ is a constant we shall fix later.

More importantly, $\gamma$ is a constant whose values characterise a family of different solutions for $(\rho(r), \chi(r))$ representing different flows to the $\mathcal{N}=2$ gauge theory in the IR. (See figure 2, on page 18.)

To date, it has been hard to extract the physics of the $\mathcal{N}=2$ gauge theory from this solution. The authors of 13, 15 have proposed that the solutions with $\gamma \leq 0$ describe the gauge theory at different points on moduli space with the $\gamma=0$ curve describing the singular point on moduli space where the gauge coupling diverges. The functional dependence of the gauge coupling on moduli space has not been reproduced so far, and we present it in this paper. For these solutions, the five dimensional supergravity potential is bounded above by the asymptotic UV value, and this is suggested in ref. [13 as a phenomenological criterion for physical acceptability.

Strong evidence for the above interpretation of the $\gamma=0$ flow was obtained in ref.[17]. There, the five dimensional criterion of ref. [13], applied to solutions in the theory perturbed to $\mathcal{N}=1$ gauge theory shows that the $\gamma=0$ solution is distinguished, as it yields the vacuum which is not lifted after the $\mathcal{N}=1$ perturbation.

It would be nice to have a fully ten-dimensional criterion for what constitutes a physical flow, and our probe analysis of this paper is a concrete step in this direction. In the calculations we present below, the physical function describing the running of the gauge coupling will emerge naturally from the solutions, and we will confirm the above identification of these solutions. 


\section{The Gauge Theory}

The $\mathcal{N}=4$ supersymmetric Yang-Mills theory's gauge multiplet has bosonic fields $\left(A_{\mu}, X_{i}\right), i=1, \ldots, 6$, where the scalars $X_{i}$ transform as a vector of the $S O(6) \mathrm{R}$-symmetry, and fermions $\lambda_{i}, i=1, \ldots, 4$ which transform as the 4 of the $S U(4)$ covering group of $S O(6)$.

In $\mathcal{N}=1$ language, there is a vector supermultiplet $\left(A_{\mu}, \lambda_{4}\right)$, and three chiral multiplets made of a fermion and a complex scalar $(k=1,2,3)$ :

$$
\Phi_{k} \equiv\left(\lambda_{k}, \phi_{k}=X_{2 k-1}+i X_{2 k}\right) \text {. }
$$

In this language, the $\mathcal{N}=2$ supersymmetric Yang-Mills theory has the vector multiplet and one massless chiral multiplet, which we can choose to be $\Phi_{3}$. The flow from the $\mathcal{N}=4$ theory to the $\mathcal{N}=2$ theory is therefore achieved by turning on operators which correspond to giving a mass to the other multiplets. As one falls well below the scale of these masses - by going to the IR - the theory becomes more effectively the theory we seek (although one must always remember that the gauge coupling is strongly coupled in the far UV in the cases where the supergravity is valid).

The operators are switched on in supergravity by considering a combination of the operators [13, 15, 16]:

$$
\begin{array}{ll}
\alpha: & \quad \sum_{i=1}^{4} \operatorname{Tr}\left(X_{i} X_{i}\right)-2 \sum_{i=5}^{6} \operatorname{Tr}\left(X_{i} X_{i}\right) \\
\chi: & \operatorname{Tr}\left(\lambda_{1} \lambda_{2}+\lambda_{2} \lambda_{2}\right)+\text { h.c. }
\end{array}
$$

The dictionary of the AdS/CFT correspondence assigns two specific scalars $(\rho=\exp (\alpha), \chi)$ to these operators. The IR/UV property [29] of the AdS/CFT correspondence then requires a non-trivial solution for these fields, making them functions of $r$ varying along the flow from the UV $(r=+\infty)$ to the IR $r=-\infty$. Consistency of the supergravity equations of motion require that there be a non-trivial back-reaction on the geometry, giving a deformation of the spacetime metric, represented by $A(r)$, etc., in section 2 . 
Moving around on the accessible part of the Coulomb branch of the $\mathcal{N}=2$ theory corresponds to giving a vacuum expectation value (vev) to $\phi_{3}=X_{5}+i X_{6}$. We shall explore this branch by moving a probe brane in those directions, and examining its physics. In the supergravity solution written in section 2, this corresponds to moving in the $(r, \phi)$ plane, setting $\theta=\pi / 2$. Attempting to move the probe out of this plane induces a nontrivial potential for the brane's motion indicating that such motion is not a modulus of the field theory. In fact, in the field theory it would correspond to inducing vevs for the massive scalars which is energetically disfavoured.

The Coulomb branch of the moduli space of the $\mathcal{N}=2 S U(N)$ gauge theory is parameterised by the vevs of the complex adjoint scalar $\phi_{3}$ which set the potential $\operatorname{Tr}\left[\phi_{3}, \phi_{3}^{\dagger}\right]^{2}$ to zero. This generically breaks the theory to $U(1)^{N-1}$. This moduli space is of course an $N-1$ complex dimensional space. The low energy effective action of the theory is described in terms of the $N-1$ complex low energy fields $\phi_{a}$, with a matrix of couplings and $\theta$-angles given in terms of the complex couplings $\tau^{a b}\left(\phi_{a}\right)$ [30, 31, 32, 33, 34]. The theory is invariant under an $S p(2 N-2, \mathbb{Z})$ group of duality transformations which shifts and inverts the coupling matrix $\tau$, and exchanges the fields $\phi_{a}$ with dual fields $\phi_{a}^{D}$. This operation, which includes a strong-weak coupling duality, is crucial in the study of the physics of the Coulomb branch, as first demonstrated in ref. 31.

We have only a one complex dimensional subspace of the moduli space here. As such, we have one complex field whose vev we wish to study and one complex coupling $\tau$ which depends on this vev. We should expect that there is a subgroup of the $\operatorname{Sp}(2 N-2, \mathbb{Z})$ duality acting on our low energy theory, and we need not look far for its origin: Our moduli space is the physics of a single D3-brane probe, moving in the $\theta=\pi / 2$ plane, and there is a $U(1)$ gauge theory living on it which shall be our effective low energy theory. There is a gauge coupling $g_{\mathrm{YM}}^{2}$ and a $\theta$-angle $\Theta$, which combine into 
a natural complex coupling

$$
\tau=\frac{\Theta}{2 \pi}+i \frac{4 \pi}{g_{\mathrm{YM}}^{2}}
$$

There is a natural $S L(2, \mathbb{Z})$ invariance of the world-volume action of the probe [35, 36, 37, which is the self duality symmetry of the type IIB superstring theory. This duality inverts and shifts the complex type IIB coupling, which is the scalar $\lambda$ described in section 2, according to

$$
\lambda \rightarrow \frac{a \lambda+b}{c \lambda+d} ; \quad a d-b c=1 \quad\{a, b, c, d\} \in \mathbb{Z} .
$$

It leaves the ten dimensional Einstein metric and the $\mathrm{R}-\mathrm{R}$ four-form invariant, and mixes the NS-NS and the R-R two-forms, under which the fundamental string and D1-brane are charged. The resulting $(p, q)$ bound state strings 38, 26] will play a role later. On the world-volume of the brane, the duality exchanges the gauge field $F_{a b}$ with a dual gauge field, $F_{a b}^{D} \equiv-2\left(\delta S / \delta F^{a b}\right)$, where $S$ is defined in equation (14).

This $S L(2, \mathbb{Z})$ action descends to an action on the effective low energy theory which we shall derive on the probe, with complex coupling $\tau$, which we will see the first hints of below. This duality transformation which acts on our moduli space, and it will be exciting to explore its uses further: It ought to give weakly coupled dual descriptions of the physics near the enhançon, where as we shall see, $g_{\mathrm{YM}}$ diverges.t A more thorough exploration of this descendant of $S L(2, \mathbb{Z})$ duality shall be left for future work.

\section{Probing with a D3-brane}

The uplifted geometry presented in ref. [15] and listed in section 2 is given in the Einstein frame. It therefore makes sense to write the D3-brane world-

\footnotetext{
${ }^{4}$ The issue of the search for a weakly coupled effective description of the enhançon is a matter discussed in ref. 40.
} 
volume action in terms of this:

$$
S=-\tau_{3} \int_{\mathcal{M}_{4}} d^{4} \xi \operatorname{det}^{1 / 2}\left[G_{a b}+e^{-\Phi / 2} \mathcal{F}_{a b}\right]+\mu_{3} \int_{\mathcal{M}_{4}}\left(C^{(4)}+\frac{1}{2} C_{(0)} \mathcal{F} \wedge \mathcal{F}\right),
$$

where $\mathcal{F}_{a b}=B_{a b}+2 \pi \alpha^{\prime} F_{a b}$, and $\mathcal{M}_{4}$ is the world-volume of the D3-brane, with coordinates $\xi^{0}, \ldots, \xi^{3}$. The parameters $\mu_{3}$ and $\tau_{3}$ are the basic $39 \mathrm{R}-\mathrm{R}$ charge and tension of the D3-brane: $\mu_{3}=\tau_{3} g_{s}=(2 \pi)^{-3}\left(\alpha^{\prime}\right)^{-2}$. Also, $G_{a b}$ and $B_{a b}$ are the pulls-back of the ten dimensional metric (in Einstein frame) and the NS-NS two-form potential, respectively. The String frame metric is $\widetilde{G}_{\mu \nu}=e^{\Phi / 2} G_{\mu \nu}$, and the pull-back is e.g.:

$$
G_{a b}=G_{\mu \nu} \frac{\partial x^{\mu}}{\partial \xi^{a}} \frac{\partial x^{\nu}}{\partial \xi^{b}}
$$

We will work in static gauge and partition the spacetime coordinates, $x^{\mu}$, as follows: $x^{i}=\left\{x^{0}, x^{1}, x^{2}, x^{3}\right\}$, and $y^{m}=\left\{r, \theta, \phi, \varphi_{1}, \varphi_{2}, \varphi_{3}\right\}$. (The $\varphi_{i}$ are angles on the deformed $S^{3}$ of section 2.) Static gauge is chosen as:

$$
x^{0} \equiv t=\xi^{0}, \quad x^{i}=\xi^{i}, \quad y^{m}=y^{m}(t) .
$$

Focusing on the subspace $\theta=\pi / 2$, for the $\mathcal{N}=2$ theory's Coulomb branch (as discussed in the previous section) we get the following result for the effective Lagrangian for the slowly moving probe moving in the transverse directions $y^{m}=(r, \phi)$ (we restrict ourselves to considering $F_{a b}=0$ here):

$$
\mathcal{L}=\frac{\tau_{3}}{2} \Omega^{2} e^{2 A} \sum_{m} G_{m m} \dot{y}^{m} \dot{y}^{m}
$$

We have neglected terms higher than quadratic order in the velocities. There is no potential term; it vanished due to a cancellation between the leading term in the expansion of the determinant, and the term containing $C^{(4)}$. This result is consistent with the fact that we have eight supercharges. (See footnote 3.) Writing this out explicitly, we see that:

$$
\mathcal{L}=\frac{\mu_{3}}{g_{s}}\left(\frac{1}{2} \Omega^{4} e^{2 A} \dot{r}^{2}+\frac{L^{2}}{2} \frac{\Omega^{4} e^{2 A}}{\rho^{8}} \dot{\phi}^{2}\right) .
$$


Next, we should attempt to find good coordinates which are appropriate to the description of the physics of the Coulomb branch of an $\mathcal{N}=2$ gauge theory.

\section{The Coulomb Branch in the UV limit}

Now before proceeding further, we should check that our result makes sense in the UV limit $(r \rightarrow \infty)$. There, we have $\Omega^{4} \rightarrow 1$, from (阿) and defining

$$
r=L \log \left(u \frac{\alpha^{\prime}}{L}\right), \text { for } L=\alpha^{1 / 2}\left(2 g_{\mathrm{YM}}^{2} N\right)^{1 / 4},
$$

where $u$ has dimensions of energy, we have:

$$
\mathcal{L}=\frac{1}{8 \pi^{2} g_{\mathrm{YM}}^{2}}\left(\dot{u}^{2}+u^{2} \dot{\phi}^{2}\right) .
$$

This is the right result. We are looking at the result for a single D3-brane probing a two dimensional subspace of the full six dimensional transverse direction to $N$ other branes. The full result is flat, as it should be for the $\mathcal{N}=4$ theory. Interpreted in terms of gauge theory, $u$ is an energy scale, or the modulus of the vev of $\phi_{3}$. We see that there is no running for $g_{\mathrm{YM}}$; there is a flat metric on moduli space.

We can write our result in the expected form [30, 31]:

$$
d s^{2} \sim \operatorname{Im} \tau d U d \bar{U},
$$

showing that the complex plane $(u, \phi)$ constitutes a good choice of coordinates for the $\mathcal{N}=2$ Coulomb branch in the UV. We shall have to work harder to find such good coordinates in the IR, as is clear from the general expression in equation (18). 


\section{The Coulomb Branch in the IR limit}

Let us use the same radial variable $u$ as we did before, initially, although we will define another shortly. The idea should be that we simply see a deviation from the flat metric (20) as we come into smaller $u$.

Since in the case $\theta=\pi / 2$ we have:

$$
\Omega^{4}=\rho^{4} \cosh 2 \chi ; \quad e^{2 A}=\frac{k^{2} \rho^{4}}{\sinh ^{2}(2 \chi)},
$$

we can simply write the moduli space metric as

$$
\begin{aligned}
d s^{2} & =\frac{L^{2} \Omega^{4} e^{2 A}}{8 \pi^{2} g_{\mathrm{YM}}^{2} u^{2} \rho^{8} \alpha^{\prime 2}}\left(\rho^{8} d u^{2}+u^{2} d \phi^{2}\right) \\
& =\frac{1}{8 \pi^{2} g_{\mathrm{YM}}^{2}} \frac{L^{2}}{\alpha^{\prime 2}} \frac{k^{2} \cosh (2 \chi)}{u^{2} \sinh ^{2}(2 \chi)}\left(\rho^{8} d u^{2}+u^{2} d \phi^{2}\right) .
\end{aligned}
$$

Our next goal is to try to understand the physics of this metric for arbitrary distance into the IR. It is convenient to define a new radial parameter, $v=u / h$, such that $h(u) \rightarrow 1 / a$ as $u \rightarrow \infty$, where $a$ is a dimensionless con-

stant. Furthermore, if we choose $\rho^{4}(u)=h(u) d v / d u$, our metric takes the simple form:

$$
d s^{2}=\frac{1}{8 \pi^{2} g_{\mathrm{YM}}^{2}} \frac{L^{2}}{\alpha^{\prime 2}} \frac{k^{2} \cosh (2 \chi)}{v^{2} \sinh ^{2}(2 \chi)}\left(d v^{2}+v^{2} d \phi^{2}\right) .
$$

In this form we can interpret the function of $v$ outside the canonical Lagrangian as the running of the coupling $\operatorname{Im} \tau$ in the $\mathcal{N}=2$ theory. The next steps are to study the behaviour of the metric component:

$$
G_{v v}(v) \equiv \frac{1}{8 \pi^{2} g_{\mathrm{YM}}^{2}(v)}=\frac{N}{4 \pi^{2}} \frac{k^{2} \cosh (2 \chi(v))}{L^{2} v^{2} \sinh ^{2}(2 \chi(v))}
$$

as a function of $v$, and also to find a suitable 30 local presentation of the effective action at least at low energy. Note now that $\chi(v)$ satisfies a different

\footnotetext{
${ }^{5}$ We thank Rob Myers for a crucial comment.
} 
differential equation. Since

$$
\frac{d \chi}{d r}=\frac{d \chi}{d u} \frac{d u}{d r}=\frac{d \chi}{d v} \frac{d v}{d u} \frac{u}{L}=\frac{d \chi}{d v} \rho^{4} \frac{v}{L},
$$

a new equation may be deduced from (9):

$$
\frac{d \chi}{d v}=-\frac{1}{2 v} \sinh (2 \chi)
$$

remarkably, this is an easy equation to solve, and the result is:

$$
\chi(v)=\frac{1}{2} \log \left[\tanh \left(\frac{1}{2} \log \left(\frac{v}{v_{e}}\right)\right)\right] .
$$

where $v_{e}$ is an integration constant!

The truly remarkable thing is that it is easy to see that the function $G_{v v}(v)$ has a zero on the circle $v=v_{e}$, which deserves to be called the "enhançon", since 23 the probe's tension is going to zero there!

(Note also that $\chi(v)$ has a mild divergence at $v=v_{e}$, and is smooth and exists for $v>v_{e}$.) The behaviour of $G_{v v}(v)$ is displayed in figure 1. (There, we have chosen various constants to be unity for plotting convenience.) The first thing to notice is that our function correctly goes to unity in the UV limit, rapidly becoming nearly constant, showing the (near) scale invariance of the theory. It fact, this nearly conformal behaviour dominates much of the flow, which is interesting. More engaging behaviour can be seen in the neighbourhood of $v=v_{e}$, where we may expand the function to see that in that region (we fix $k=2 v_{e} \alpha^{\prime} /(a L)$ by examining the UV asymptotics and also equation (22)):

$$
d s^{2} \sim N \log \left(\frac{v}{v_{e}}\right)\left(d v^{2}+v^{2} d \phi^{2}\right) .
$$

which is the one-loop logarithmic running we expect from the gauge coupling of a $U(1)$ low energy theory on the Coulomb branch of the $\mathcal{N}=2$ gauge theory!

(It is important to note that we have set $a^{-2}=g_{\mathrm{YM}}^{2} N$ by hand. One would expect to be able to fix $a$ uniquely in terms of the supergravity parameters in 

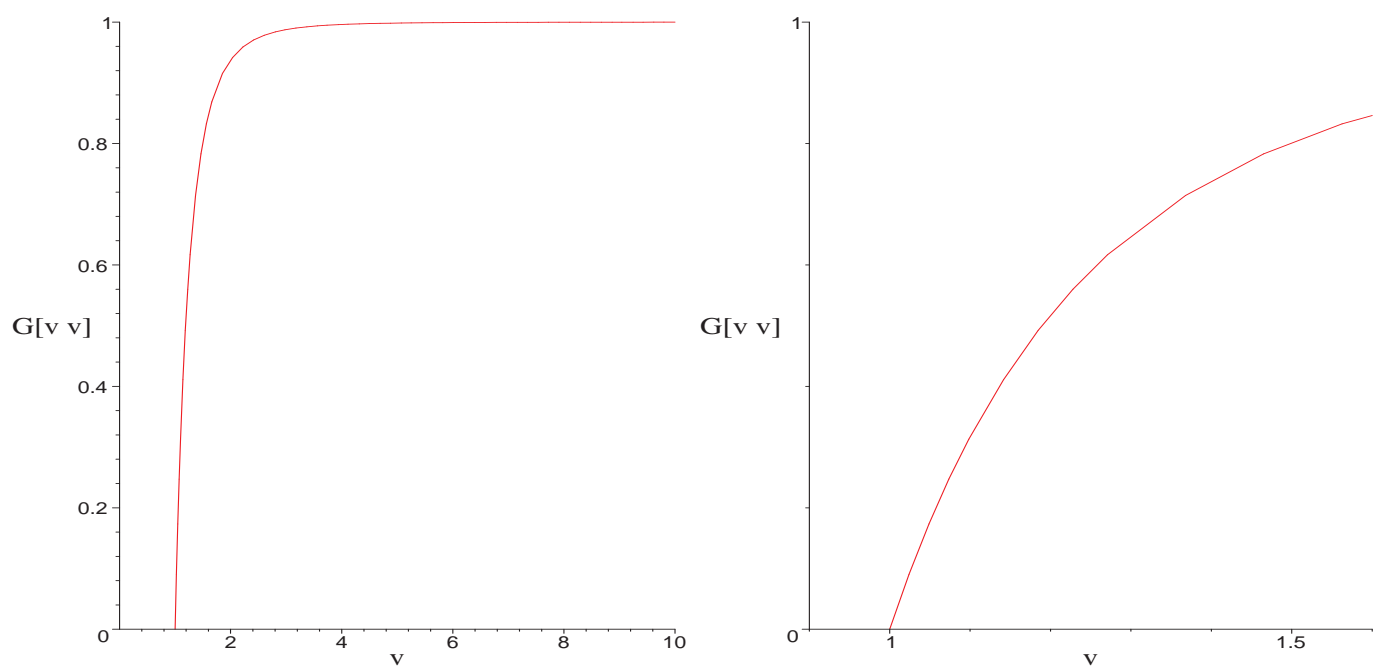

Figure 1: The metric function $G_{v v}$ representing the moduli space probe result for the D3-brane. It is proportional to the probe's tension. The enhançon is at $v_{e}=1$ here. This is also the result for the running of the inverse squared Yang-Mills gauge coupling as a function of energy scale $v$. It stops running in the UV. On the right is a closeup, showing the logarithmic running near $v_{e}$, in the IR.

the solution, and that we have not quite done this suggests that our attempt to adjust the conventions of ref. 15 to restore all factors of $N$ and $g_{\mathrm{YM}}$ is incomplete. However, we are confident that our parameters are correct for the following reasons: On general grounds [19], the complete decoupling from gauge theory of the dual supergravity ensures that it must depend on $g_{\mathrm{YM}}$ and $N$ only through the t'Hooft coupling $\lambda=g_{\mathrm{YM}}^{2} N$. So our choice for $a$ is not unnatural, up to pure numbers. Furthermore, the structure of the AdS/CFT correspondence is such that we should get the one-loop field theory result in this way, which fixes the factor of $g_{\mathrm{YM}}$, and then the $N$ must accompany it to make $\lambda$. See also ref.[23].)

Quite pleasingly, we have succeeded in showing analytically that our metric (and hence our low energy effective action) can be written in the form:

$$
d s^{2} \sim \operatorname{Im} \tau(V) d V d \bar{V}
$$


where now the complex coordinate $V$ is the $(v, \phi)$ plane. ${ }^{0}$ The full metric interpolates between this form of coordinates in the IR and the similar form (21) found earlier in terms of the the coordinate $U$ in the UV.

We are now ready to discuss the interpretation and consequences of our computation for the Coulomb branch of the gauge theory.

\section{Families of Flows: The Moduli Space}

As mentioned before, the $\mathcal{N}=2$ flows are characterised into three different classes $\gamma<0, \gamma=0$ and $\gamma>0$ (see figure 2, on page 18). Equation (27) and the metric (28) must be interpreted carefully for each of these flows.

Let us first check the supergravity behaviour of these flows:

- For $\gamma<0$, equation (10) yields a finite value of $\chi$ in the IR, while $\rho$ goes to zero. It is clear, from the functions listed at the beginning of section 2, that the ten dimensional supergravity solution has a naked singularity as a result.

- For $\gamma=0, \chi$ diverges in the IR and again $\rho$ goes to zero. Supergravity again has singular behaviour, coming from both the divergence and the zero.

- For $\gamma>0$ both $\chi$ and $\rho$ diverge, and the supergravity is singular.

Let us now see how this behaviour combines with our equations (27) and (28) to give sensible physics.

\footnotetext{
${ }^{6}$ Added in v2 of preprint: One can check that our function agrees with the coefficient of the $F^{2}$ term in the effective action on the probe. That coefficient is simply $e^{-\Phi}$ and from (27) and (32), it can be seen that its IR limit is also proportional to $\log \left(v / v_{e}\right)$. The dependence of the coefficient on $\phi$ can be absorbed by a rescaling of $v$. There is $\phi$ dependence in the coupling since the $U(1)$ symmetry on the $\mathcal{N}=2$ theory's moduli space is not an exact symmetry of the broken $\mathcal{N}=4$ theory, especially when, as in this case, the $\mathcal{N}=4$ matter is not decoupled at the strong coupling scale. See ref.42 for a change of coordinates which emphasises this, while converting the circular enhançon into a line segment by squashing it.
} 


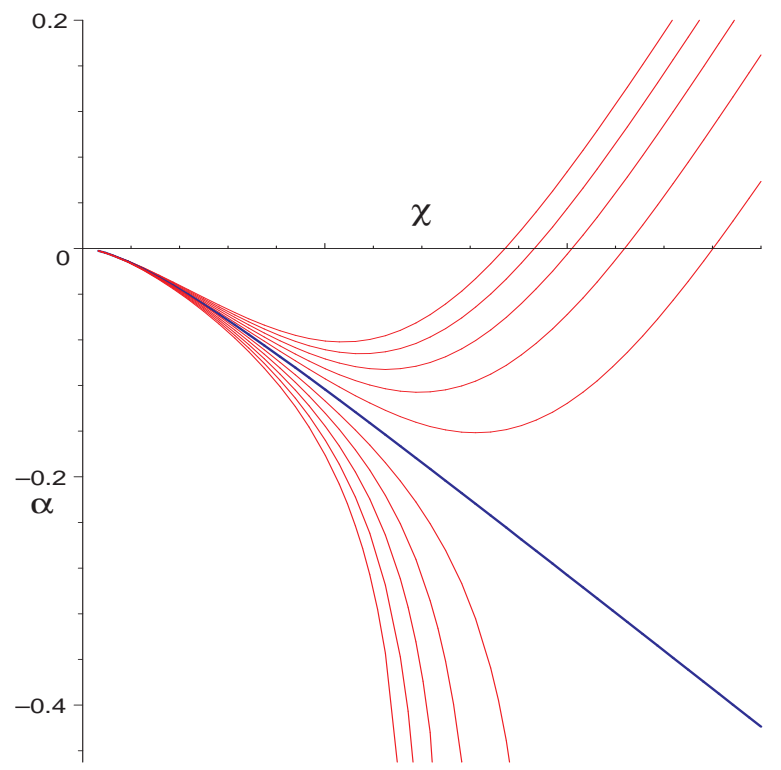

Figure 2: The families of $(\chi, \alpha)$ curves for differing $\gamma$, given by equation (10). There are three classes of curves. The blue (middle) curve is $\gamma=0$, the $\gamma<0$ curves are below it, and the $\gamma>0$ curves are above. The flow from UV to IR along each curve is to the right. Recall that $\rho=e^{\alpha}$, and refer to the text for further physical interpretation of each curve.

- For the $\gamma<0$ solutions, $\rho \rightarrow 0$ in the IR while $\chi$ approaches some fixed value, $\tilde{\chi}$, different from zero. From equation (27), this translates into some specific value of $v>v_{e}$, which we shall denote as $w$. This is a particular point (or ring of points) on the moduli space, and although supergravity diverges there, we have the right to ask physical questions. This is where the probe physics comes in: The metric that the probe sees is perfectly smooth at $w$. We can evaluate the gauge theory coupling as seen by the probe there using (28) even though supergravity is singular. Deep enough in the IR, the result is proportional to $N \log \left(w / v_{e}\right)$, just as it should be for the low energy effective gauge theory, at position/vev $w$ on the moduli space. 
- For the $\gamma=0$ solutions, $\chi$ diverges, and this is at $v=v_{e}$. Supergravity has diverged and we appeal again to the physics of the probe to help us. We see that the tension of the probe drops to zero there: the gauge coupling diverges logarithmically as seen in equation (28). This is the enhançon, and the radius $v=v_{e}$ is the locus of points where there are new massless degrees of freedom in the gauge theory.

We should digress here. The latter fact is borne out by the realisation that the mass of a particle made by a macroscopic string stretched orthogonally to the D3-branes along the $r$ direction (which make dyonic bound states in the gauge theory) has a chance to drop to zero at $v=v_{e}$, since (by an analogous computation to that done for the D3-brane) it is proportional to

$$
\mathcal{E}=\int_{r}^{r_{e}} d r F(r)\left(G_{r r} G_{t t}\right)^{1 / 2}=\int_{r}^{r_{e}} d r F(r) \frac{\cosh ^{1 / 2}(2 \chi)}{\sinh (2 \chi)} k \rho^{4} .
$$

The part of the integrand not involving $F(r)$ is going to zero, as its square is proportional to the D3-brane probe's $G_{v v}$. The prefactor function $F(r)$ depends upon the type of macroscopic string under investigation. For the D-string, $F_{0,1}(r)=\left(2 \pi \alpha^{\prime}\right)^{-1} e^{-\Phi / 2}$, but it can be more complicated for other strings, since their tension is controlled by [38]:

$$
F_{p, q}(r)=\frac{1}{2 \pi \alpha^{\prime}}\left[e^{\Phi}\left(q C_{(0)}-p\right)^{2}+q^{2} e^{-\Phi}\right]^{1 / 2} .
$$

Inserting this into equation (30) essentially yields a formula for the mass of a $(p, q)$ dyon in the gauge theory as a function of $v$, as it is made of a bound state of $p$ fundamental strings and $q$ D1-branes ending on the D3-branes, whose tension is given by (30). These dyons will be mapped into one another by the $S L(2, \mathbb{Z})$ duality group bequeathed to the low energy theory by the parent type IIB string theory.

Given that we are in a non-trivial background, there is the possibility that divergent enough $F(r)$ might appear, to save some bound states from 
going massless, and it is interesting to compute just which of the $(p, q)$ do, generalising the structures in ref. [31]. In fact, (for the moduli space choice $\theta=\pi / 2$ ), equations (6) and (17) yield:

$$
\begin{aligned}
e^{-\Phi} & =\frac{2 \cosh (2 \chi)}{\cosh ^{2}(2 \chi)(1+\cos 2 \phi)+1-\cos 2 \phi}, \text { and } \\
C_{(0)} & =\frac{\sinh ^{2}(2 \chi) \sin 2 \phi}{\cosh ^{2}(2 \chi)(1+\cos 2 \phi)+1-\cos 2 \phi} .
\end{aligned}
$$

Furthermore, taking the limit appropriate to $\gamma=0$, (i.e., $\chi \rightarrow \infty$ and $\rho \rightarrow 0$ ), we see that because of the large power of $\rho$ present in equation (30), all of the types of strings/dyons are tensionless on the enhançon, since nothing can save $\mathcal{E}(r)$ from going to zero as $r \rightarrow r_{e}$. This is generalisation of the features in ref. 31 which is consistent with the fact that constituent D3-branes of the supergravity solution have spread out to form this ring, and so all strings stretched between our probe and the background D3-branes will give zero mass states in the limit.

A tantalising feature of the special family of vacua represented by the enhançon is that the dilaton and $\mathrm{R}-\mathrm{R}$ scalar change as one goes around the circle in $\phi$, moving along the enhançon ring: the $(p, q)-$ "flavour" of the vacuum changes! Since the overall factor not involving $F_{p, q}(r)$ is common to all, it seems intuitively clear that there is a "lightest" dyon state among those which go massless, which characterises the vacuum at a given value of $\phi$. This is easy to see by plotting some samples of the behaviour of $F_{p, q}(\phi)$ for a value of $v$ away from the enhançon, as we have done in figure 3 .

Furthermore, it is clear that the duality which descends from the $S L(2, \mathbb{Z})$ of the type IIB string plays a beautiful role here, by exchanging the different vacua at different points on the circle. For example, the points $\phi=$ $\{0, \pi / 2, \pi, 3 \pi / 2\}$, forming the principal points on the compass, are special in that $C_{(0)}=0$. So a $(p, q)$ dyon's mass is controlled by the familiar Pythagorean form: $\sqrt{p^{2} e^{\Phi}+q^{2} e^{-\Phi}}$. However, as we go around the four 

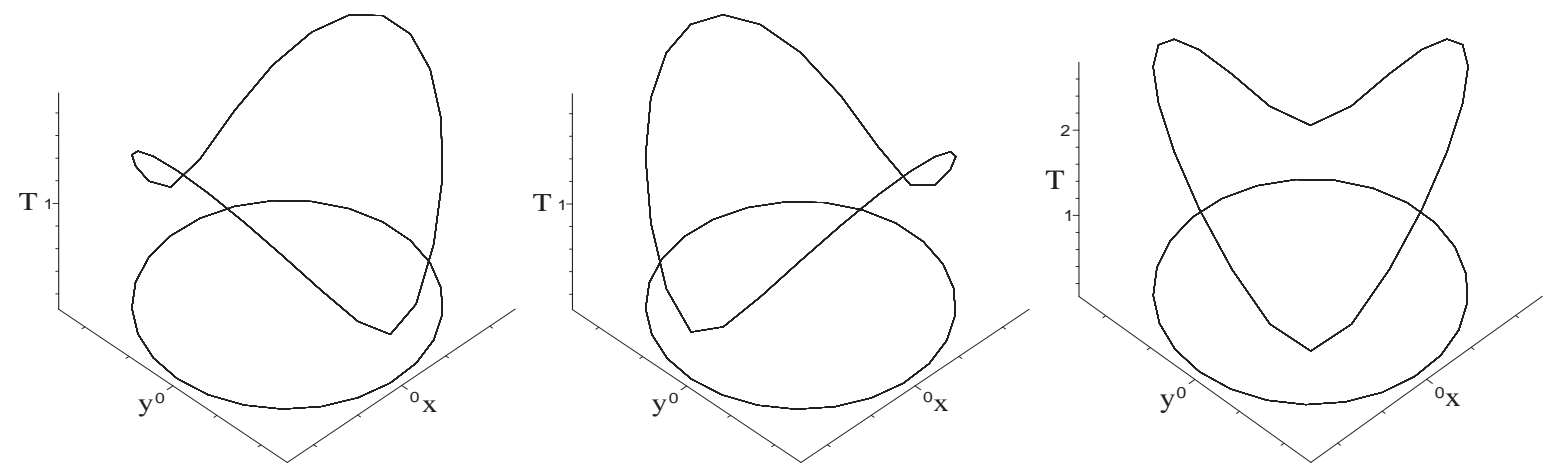

Figure 3: The functional dependence of the tension of $(1,0),(0,1)$, and $(1,1)$ strings as one goes around a generic circle. At the enhançon, any point on the circle will have a lightest string/dyonic state.

points, the dilaton toggles between $\Phi$ and $-\Phi$. These are clearly vacua which are related by the action of the $S$ generator of the $S L(2, \mathbb{Z})$. We should now return to our discussion of the flows.

- The $\gamma>0$ curves appear to give behaviour which is irretrievably unphysical from both the point of view of the supergravity and the probe. This conclusion is in line with the previous suggestions of refs. [15, 13, 16], but we make a more detailed suggestion: In it natural to suppose that these flows attempt to take us behind the enhançon ring to get to the limiting IR physics, where the tension of the probe would need to be (unphysically) negative in order to preserve supersymmetry. We propose, in the spirit of ref. [23], that these are not physical solutions for that region. Excising that part of the supergravity solution and (perhaps) replacing it with flat space seems prudent. Whether or not this gives a description of a part of gauge theory moduli space possibly hidden inside the enhançon is a matter for future work.

We note again that we also have a full strong/weak coupling duality group acting on the low energy gauge theory. (It is inherited from the $S L(2, \mathbb{Z})$ 
symmetry of the D3-brane within type IIB string theory, as discussed in section (3). This means that although the gauge coupling diverges on the probe at the enhançon, there is a dual description to a low energy theory with coupling $1 / \tau$ and complex modulus field $V_{D}$. This duality may be interesting to study, although the dual vacua reached thus will also have a full tower of massless dyons, and may require altogether new techniques to study them, since some non-locality certainly arises. Nevertheless, this might be a promising new handle on the issue 40] of finding an effective lowenergy description of the physics near the enhançon, and may also shed light on whether one can make sense of the stringy physics behind it in terms of gauge theory.

\section{Added Note}

We have been informed [41] that Alex Buchel, Amanda Peet, and Joe Polchinski have done independent work on the issue of probing the solution of ref. [15], and understanding the role of the enhançon in this context [42].

\section{Acknowledgements}

We thank Juan Maldacena, Rob Myers, Joe Polchinksi, Nick Warner and Alberto Zaffaroni for conversations and comments. N.E. is grateful to PPARC for the sponsorship of an Advanced Fellowship. C.V.J. would like to thank the Aspen Centre for Physics for hospitality while this work was completed. M.P. is partially supported by INFN and MURST, and by the European Commission TMR program ERBFMRX-CT96-0045, wherein she is associated to Imperial College, London, and by the PPARC SPG grant 613. This paper is report number DTP/00/69 at the CPT, Durham. 


\section{References}

[1] L. Girardello, M. Petrini, M. Porrati and A. Zaffaroni, JHEP 9812 (1998) 022, hep-th/9810126.

[2] J. Distler and F. Zamora, Adv. Theor. Math. Phys. 2 (1998) 1405, hepth/9810206.

[3] A. Khavaev, K. Pilch and N. P. Warner, "New Vacua of Gauged N=8 Supergravity", hep-th/9812035.

[4] A. Karch, D. Lüst and A. Miemiec, Phys. Lett. B454 (1999) 265, hepth/9810254.

[5] S. Nojiri and S. D. Odintsov, Phys. Lett. B449, 39 (1999), hepth/9812017; Phys. Rev. D61, 044014 (2000), hep-th/9905200.

[6] A. Kehagias and K. Sfetsos, Phys. Lett. B454 (1999) 270,hepth/9902125; S. S. Gubser, "Dilaton-driven confinement", hepth/9902155; N. R. Constable and R. C. Myers, JHEP 9911 (1999) 020, hep-th/9905081.

[7] L. Girardello, M. Petrini, M. Porrati and A. Zaffaroni, JHEP 9905 (1999) 026, hep-th/9903026.

[8] D. Z. Freedman, S. S. Gubser, K. Pilch and N. P. Warner, "Renormalization Group Flows from Holography-Supersymmetry and a c-Theorem", hep-th/9904017.

[9] D. Z. Freedman, S. S. Gubser, K. Pilch and N. P. Warner, "Continuous distributions of D3-branes and gauged supergravity", hep-th/9906194;

[10] A. Brandhuber and K. Sfetsos, "Wilson loops from multicentre and rotating branes, mass gaps and phase structure in gauge theories", hepth/9906201; I. Chepelev and R. Roiban, Phys. Lett. B462 (1999) 74, 
hep-th/9906224; I. Bakas and K. Sfetsos, Nucl. Phys. B573 (2000) 768, hep-th/9909041.

[11] L. Girardello, M. Petrini, M. Porrati and A. Zaffaroni, Nucl. Phys. B569 (2000) 451, hep-th/9909047.

[12] J. Distler and F. Zamora, JHEP 0005 (2000) 005, hep-th/9911040

[13] S.S. Gubser "Curvature Singularities: the Good, the Bad, and the Naked", hep-th/0002160.

[14] M. Petrini and A. Zaffaroni, "The Holographic RG flow to conformal and non-conformal theory", hep-th/0002172.

[15] K. Pilch and N. P. Warner, "N=2 Supersymmetric $R G$ Flows and the IIB Dilaton", hep-th/0004063.

[16] A. Brandhuber and K. Sfetsos, "An N=2 gauge theory and its supergravity dual", hep-th/0004148.

[17] N. Evans and M. Petrini, "AdS RG-Flow and the Super-Yang-Mills Cascade", hep-th/0006048.

[18] K. Pilch and N. P. Warner, "N=1 Supersymmetric Renormalization Group Flows from IIB Supergravity", hep-th/0006066.

[19] J. Maldacena, Adv. Theor. Math. Phys. 2 (1998) 231, hep-th/9711200.

[20] S.S. Gubser, I.R. Klebanov and A.M. Polyakov, Phys. Lett. B428 (1998) 105, hep-th/9802109.

[21] E. Witten, Adv. Theor. Math. Phys. 2 (1998) 253, hep-th/9802150.

[22] For a discusssion, in this context, see ref. 113. 
[23] C. V. Johnson, A. W. Peet and J. Polchinski, Phys. Rev. D61, 086001 (2000), hep-th/9911161.

[24] M. R. Douglas, J. Geom. Phys. 28, 255 (1998), hep-th/9604198.

[25] J. Polchinski, Phys. Rev. D50 (1994) 6041, hep-th/9407031.

[26] E. Witten, Nucl. Phys. B460 (1996) 335, hep-th/9510135.

[27] M. R. Douglas, D. Kabat, P. Pouliot and S. H. Shenker, Nucl. Phys. B485, 85 (1997), hep-th/9608024.

[28] C. V. Johnson, "D-Brane Primer", hep-th/0007170.

[29] L. Susskind and E. Witten, "The holographic bound in anti-de Sitter space", hep-th/9805114;

A. W. Peet and J. Polchinski, Phys. Rev. D59, 065011 (1999), hepth/9809022.

[30] N. Seiberg, Phys. Lett. B206 (1988) 75.

[31] N. Seiberg and E. Witten, Nucl. Phys. B426 (1994) 19; Erratum: ibid., B430 (1994) 485, hep-th/9407087; ibid., B431 (1994) 484, hepth/9408099.

[32] P. C. Argyres and A. E. Faraggi, Phys. Rev. Lett. 74, 3931 (1995), hep-th/9411057.

[33] A. Klemm, W. Lerche, S. Yankielowicz and S. Theisen, Phys. Lett. B344, 169 (1995), hep-th/9411048.

[34] M. R. Douglas and S. H. Shenker, Nucl. Phys. B447, 271 (1995), hepth/9503163.

[35] G. W. Gibbons and D. A. Rasheed, Phys. Lett. B365, 46 (1996), hepth/9509141. 
[36] M. B. Green and M. Gutperle, Phys. Lett. B377, 28 (1996), hepth/9602077.

[37] A. A. Tseytlin, Nucl. Phys. B469, 51 (1996), hep-th/9602064.

[38] J. H. Schwarz, Phys. Lett. B360 (1995) 13; (E) B364 (1995) 252, hepth/9508143.

[39] J. Polchinski, Phys. Rev. Lett. 75 (1995) 4724, hep-th/9510017.

[40] C. V. Johnson, "Enhançons, fuzzy spheres and multi-monopoles" hepth/0004068.

[41] J. Polchinski, private communication on June 20th;

J. Polchinski, public communication (his talk) at Strings 2000, University of Michgan, July 2000.

[42] A. Buchel, A. Peet and J. Polchinski, to appear. 